\title{
Impact of introducing integrated quality assessment for tuberculosis and malaria microscopy in Kano, Nigeria
}

\author{
Faruk Sarkinfada ${ }^{1}$, Yunusa Aliyu ${ }^{2}$, Charles Chavasse $^{3}$, Imelda Bates ${ }^{3}$ \\ ${ }^{1}$ Department of Medical Microbiology and Parasitology, Faculty of Medicine, Bayero University, Kano, Nigeria \\ ${ }^{2}$ Kano State Tuberculosis and Leprosy control Programme, Ministry of Health Kano, Nigeria \\ ${ }^{3}$ Disease Control Strategy Group, Liverpool School of Tropical Medicine, United Kingdom
}

\begin{abstract}
Background: The World Health Organization (WHO) has recommended the integration of malaria microscopy quality assessment (QA) with that of other microscopically diagnosed diseases, but there is no evidence that it has been attempted. We assessed the feasibility of linking malaria microscopy into the existing tuberculosis (TB) microscopy QA system in Kano, Nigeria.

Methodology: Five TB microscopy centres were selected for implementing the integrated TB and malaria microscopy QA scheme in the state. A model system was designed for selecting and blinded rechecking of TB and malaria slides from these laboratories. Supervision and evaluation was conducted at 3-month intervals for 24 months.

Results: TB microscopy QA was strengthened in four laboratories. Full integration of the QA for TB and malaria microscopy was achieved in two laboratories, and partial integration in two other laboratories. The programme resulted in an increase in the specificity of both TB and malaria microscopy results. At the final assessment, $100 \%$ specificity was achieved for TB microscopy results in four laboratories. There was an increased concordance rate and decreased false positivity and false negativity rates of TB microscopy results in all five laboratories.

Conclusions: It is feasible to integrate the QA system and training for TB and malaria microscopy for assessing and improving quality of both services. However, the integrated system needs testing in different settings in order to be able to develop sound recommendations to guide the complex scaling-up process.
\end{abstract}

Key Words: tuberculosis, malaria, microscopy, quality, Kano

J Infect Developing Countries 2009; 3(1):20-27.

Received 12 May 2008 - Accepted 04 September 2008

Copyright $\odot 2008$ Sarkinfada et al. This is an open access article distributed under the Creative Commons Attribution License, which permits unrestricted use, distribution, and reproduction in any medium, provided the original work is properly cited.

\section{Introduction}

Kano State is one of the 36 states of Nigeria, located in the northern part of the country. It has an estimated population of over 12 million people of which $75 \%$ live in the rural areas [1]. Tuberculosis (TB) and malaria are endemic in Nigeria and among the most important public health burdens of the country. Each year in Nigeria, 220,000 positive cases of pulmonary tuberculosis cases occur of which approximately $50 \%$ are smear-positive. The country was ranked fourth among the 22 countries with the highest burden of TB [2]. In Kano State, there were 5,179 registered cases of tuberculosis at end the of 2006, representing about $30 \%$ of the estimated number of TB cases in the state. There were 2,608,479 reported cases and 5,343 deaths due to malaria in Nigeria in 2003 [3]. There were 264,481 reported cases of malaria in Kano State in the year 2003, with prevalence of 22 per 1000 population [4].
Sputum smear microscopy for detecting acid-fast bacilli (AFB) and blood film microscopy for detecting parasites are important laboratory procedures for diagnosis and management of $\mathrm{TB}$ and malaria respectively. In order to achieve the required quality in laboratory diagnosis, a continuous system of quality assessment (QA) needs to be established [5,6]. The laboratory QA schemes used in industrialized countries are based on the assumption that the laboratory methods are generally automated and communication and transport systems are reliable, and are therefore not suitable for laboratories in low-income countries [7].

In Kano there was a network of ten designated TB microscopy laboratories supporting the Directly Observed Treatment Short Courses (DOTS) in the year 2005, and a quality assessment scheme for TB microscopy. There was no network of malaria microscopy laboratories or quality assessment scheme for malaria microscopy at any level of health care in the 
state. In contrast to the situation for $\mathrm{TB}$, the national policy for malaria does not include any QA guideline for microscopy [8]. The WHO has recommended the integration of malaria microscopy QA with that of other microscopically diagnosed communicable diseases [9], but there is no evidence for how this can be put into practice.

We conducted a baseline assessment of medical laboratory services in Kano State and designed, implemented and evaluated an integrated system for measuring and improving quality of $\mathrm{TB}$ and malaria microscopy.

\section{Materials and Methods}

Baseline assessment of medical laboratory services for $T B$ and malaria in Kano state

The design of a QA system for TB and malaria microscopy requires retrospective information on the infrastructure, workload and slide positivity rates for TB and malaria microscopy. A checklist for laboratory assessment was developed using published guidelines $[10,11]$ and checklists used in other West African countries [12]. Information on staffing and the number of TB and malaria tests conducted were collected from 13 of the 34 medical laboratories in Kano state.

The 13 selected laboratories included laboratories in the the following three largest specialist hospitals in urban Kano, where laboratory services are concentrated: Murtala Muhammad Specialist hospital (MMSH), Muhammad Abdullahi Wase Specialist Hospital (MAWSH), and Hasiya Bayero Paediatric Hospital (HBP). The following ten designated DOTS microscopy centres located in urban and semi-urban areas were also included in the study: the Infectious Disease Hospital (IDH), Wudil General Hospital (WDL) Gezawa General Hospital (GZW) Gwarzo General Hospital (GWZ), Danbatta General Hospital (DBT), Kura General Hospital (KUR), Bichi General Hospital (BCH) Rano General Hospital (RAN), Sumaila General Hospital (SML), and Karaye General Hospital (KRY). The selected laboratories were representative laboratories where laboratory services in Kano state are likely reflected.

Five out of the 10 designated microscopy centres (IDH, WDL, GWZ, RAN and DBT) where TB microscopy slides were stored were selected for implementing the integrated $\mathrm{TB}$ and malaria microscopy QA scheme. Examined TB slides were sampled from the five DOTS microscopy centres. Malaria slides were from only two of the five centres
(IDH and WDL) where malaria slides were available during the assessment period.

\section{Method of sampling slides}

The Lot Quality Assurance System (LQAS) sampling method (APHL, 2002) was used for selecting the examined TB and malaria microscopy slides. LQAS sampling determines an optimum sample size that yields a statistically acceptable sample to assess quality of work, in this case TB and malaria microscopy $[13,9]$. The numbers of TB and malaria slides selected were determined using the Lot number, which is the average number of negative TB or malaria slides examined by a laboratory in three-month period (quarter). A standard reference table of sample sizes set at a critical value of $5 \%$, sensitivity of $80 \%$, specificity of $100 \%$, acceptance number $\mathrm{d}=0$ and $95 \%$ Confidence Interval was used to determine the number of TB and malaria slides required from each laboratory for rechecking per each quarter [11]. Thus 17 TB slides were required quarterly from each laboratory based on an annual average of 5,000 negative smears per laboratory, with a positivity rate of $15 \%$. Eight malaria slides were required quarterly from each laboratory based on an annual average of 2000 negative slides per laboratory and a slide positivity rate of $47.2 \%$.

Sampling intervals were determined by dividing the total number of slides examined in the quarter by 17 and eight for TB and malaria slides respectively. The resulting number $(\mathrm{N})$ was the interval between each of the slides to be selected. In order to eliminate selection bias, slides were selected by the supervisor from the entire lot using the laboratory register irrespective of whether the result was positive or negative and regardless of the quality of the smear or staining. The selected slides were retrieved from the slide boxes with the help of the microscopists in the laboratory. If a slide was missing, it was substituted by the one after it. When several slides were missing, the selection was repeated from the register starting from a different point.

Design and implementation of integrated TB and malaria microscopy $Q A$ scheme

A group of five experienced TB and malaria microscopists were selected from the tertiary and secondary healthcare facilities in Kano to collaborate on the project. A one-day refresher training workshop on the identification of AFB in $\mathrm{ZN}$-stained sputum smear and malaria parasites in Leishman-stained thin blood film was conducted for the QA team together with the microscopists from the five selected centres. The 
purpose of the training was to demonstrate the degree of accord and discord between the original results of malaria parasites microscopy from the source centres and the results obtained from the rechecking process by the participants, and to develop an integrated, feasible method for assessment of the quality of TB and malaria smear / blood films staining. The training involved group rechecking and evaluation of $\mathrm{TB}$ slides and malaria slides from participating centres, and examination and reporting of positive and negative control TB and malaria slides.

Community tuberculosis control supervisors from the five selected centres were also trained on how to incorporate supervision of malaria microscopy and slide selection for quality assessment into the TB quality assessment scheme.

TB and malaria microscopy slides were collected by the TB supervisor and submitted to the state TB quality control officer at three-month intervals for rechecking at a state secondary laboratory. The slides were rechecked independently by two of the five selected microscopists. TB and malaria microscopy checks were integrated by using a pair of microscopists from the pool of five experienced microscopists with skills in both malaria and TB microscopy. Although the scheme focused on QA, the quality control culture in these laboratories was assessed by the availability of microscopists during supervisory visits and their ability to make available all the $\mathrm{TB}$ and malaria slides examined in their laboratories (Figure 1).

\section{Protocol for re-checking of TB and malaria slides}

The first reader re-examined the sampled slides visually to assess the quality of smear and blood film preparation as good, fair or bad using predefined criteria (Table 1). One hundred high-power fields were examined for negative. Results from TB slides were graded using the standard grading table for TB microscopy [14]. Results of malaria microscopy were recorded as positive Plasmodium falciparum if trophozoites were present at $<10$ /field and $>10$ /field, or negative to indicate their absence [9].

The quality control officer compared the first reader's result with those of the original reader. If the original and first reader results agreed, the original was accepted. Slides with discordant results were counterchecked by the second reader without knowing the results of the first reader. The final result was taken as any two consensus results among the three readings. Errors in reading TB and malaria microscopy slides were classified as:
- False positives (FP), when a negative sputum smear or blood film was misread positive

- False negatives (FN), when a positive sputum smear or blood film was misread as negative.

Table 1. Criteria for assessing the quality of TB and malaria slides.

\begin{tabular}{ll}
\hline & $\begin{array}{l}\text { Quality of preparation and staining of sputum smear and } \\
\text { blood films for TB and malaria microscopy }\end{array}$ \\
\hline Grades & Sputum smear / staining* \\
\hline Good & $\begin{array}{l}\text { Smear of approximately 1 x } 2 \text { cm, evenly distributed on the } \\
\text { glass slide; thickness allows printed letters to be read through. }\end{array}$ \\
& $\begin{array}{l}\text { Staining allows AFB and the background to be clearly } \\
\text { distinguished as red against a bluish background. }\end{array}$ \\
\hline Fair & $\begin{array}{l}\text { Smear is thick so that printed letter cannot be read through or } \\
\text { unevenly distributed with a size more than 1x 2 cm. }\end{array}$ \\
& $\begin{array}{l}\text { Staining: Due to over decolourization, AFB appears faint red } \\
\text { colour or due to under decolourization remnants of carbol } \\
\text { fuchsin or methylene blue appear on the background or } \\
\text { unsmeared parts of the slide. }\end{array}$ \\
\hline Bad & $\begin{array}{l}\text { Smear is made from saliva and was too thin with indefinite } \\
\text { size or made too thick so that printed letters cannot be read } \\
\text { through. }\end{array}$ \\
Stains stick on the smear and fields difficult to spot with AFB \\
or \\
Smear was made from saliva and cannot pick up the stain.
\end{tabular}

* Technical guide for Sputum Examination for Tuberculosis by Direct Microscopy in Low Income Countries [14]. RBC $=$ Red blood cells

The assessments of quality of $\mathrm{TB}$ and malaria microscopy used were 1) the proportions of original microscopy results that were concordant, false positive or false negative; 2) the proportions of well prepared and / or stained sputum smears and blood films; 3 ) the sensitivity defined as the ability of microscopists in the study laboratories to detect AFB or malaria parasites relative to the final results, and specificity defined as the ability of microscopists in the study laboratories to identify AFB or malaria parasites correctly when 
present in sputum smears or blood films. Trends in the

Figure 1. An integrated $\mathrm{QA}$ scheme for $\mathrm{TB}$ and malaria microscopy.

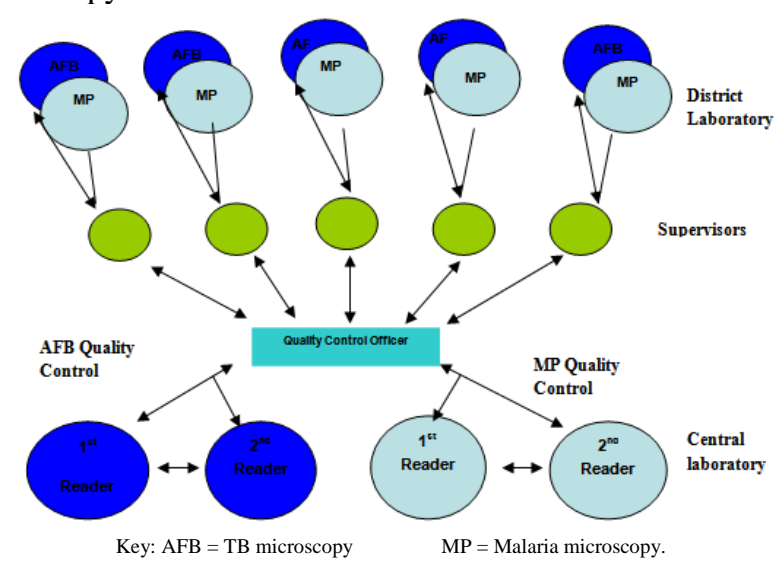

changes of quality assessment and comparison of the baseline and final performance of each laboratory were evaluated.

\section{Evaluation of integration}

Full integration of $\mathrm{TB}$ and malaria EQA in laboratories was defined as the three assessments for malaria microscopy that were conducted during the TB microscopy supervisory visits. Partial integration was defined as at least one joint assessment and no integration was where malaria microscopy assessment was not conducted.

SPSS 15.0 software was used to determine the statistical significance of the changes observed between the periods of assessment using the Chi square test of significance. Kappa statistics were used to determine the coefficient of agreement (Kappa) between results from the district laboratories and the first and second rereadings, using the Win Episcope version 2.0 programme. Correlation coefficients between the numbers of concordant results and the numbers of good, fair and bad sputum smears and/or blood films and staining were determined using the SPSS programme.

\section{Results}

Baseline assessment of $T B$ and malaria laboratory services in Kano State (April 2005)

The profiles of laboratories included in the baseline assessment are presented in Table 2. Microscopy tests made up $21 \%$ of the tests conducted in one year. The proportions of malaria and AFB microscopy among the microscopy tests were $35.1 \%$ and $27.2 \%$ respectively. The highest numbers of $\mathrm{TB}$ and malaria microscopy tests in Kano were conducted in IDH and MMSH laboratories respectively.

The five laboratories selected for implementing TB and malaria microscopy quality assessments had at least one microscope and two microscopists covering both TB and malaria. TB microscopy was concentrated at IDH, where 12,525 TB slides were examined in 2004, constituting more than $70 \%$ of all the TB microscopy slides examined in the same year. The number of TB

Table 2. Profiles of thirteen medical laboratories in Kano state.

\begin{tabular}{|c|c|c|c|c|c|}
\hline & Infrastructure & d workload & & & \\
\hline $\begin{array}{l}\text { Levels of } \\
\text { care }\end{array}$ & Laboratories & $\begin{array}{c}\text { Number of } \\
\text { rooms }\end{array}$ & $\begin{array}{c}\text { Number } \\
\text { of staff }\end{array}$ & $\begin{array}{c}\text { Number of } \\
\text { tests } \\
\text { conducted in } \\
2004\end{array}$ & $\begin{array}{c}\begin{array}{c}\text { Number } \\
\text { of } \\
\text { microsco } \\
\text { pes }\end{array} \\
\end{array}$ \\
\hline \multirow{2}{*}{ Tertiary } & MMSH & 7 & 60 & 196392 & 3 \\
\hline & MAWSH & 5 & 17 & 49159 & 3 \\
\hline \multirow{11}{*}{ Secondary } & HBP & 2 & 8 & 4821 & 2 \\
\hline & IDH* & 2 & 6 & 19244 & 1 \\
\hline & WDL* & 1 & 5 & 25481 & 1 \\
\hline & GZW* $^{*}$ & 1 & 3 & 7454 & 1 \\
\hline & GWZ* & 1 & 5 & 5465 & 1 \\
\hline & DBT* & 1 & 6 & 16768 & 1 \\
\hline & KUR* & 1 & 5 & 7086 & 1 \\
\hline & BCH $^{*}$ & 1 & 5 & 5202 & 1 \\
\hline & RAN* & 1 & 4 & 8975 & 1 \\
\hline & SML* & 1 & 3 & 3426 & - \\
\hline & KRY* & 1 & 1 & 264 & - \\
\hline Total & 13 & & 128 & 287786 & 16 \\
\hline $\begin{array}{l}\text { Levels of } \\
\text { care }\end{array}$ & $\begin{array}{c}\text { Number of } \\
\text { TB/malaria } \\
\text { microscopists }\end{array}$ & 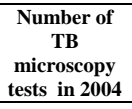 & $\begin{array}{l}\text { TB slide } \\
\text { positivity } \\
\text { rate }(\%)\end{array}$ & $\begin{array}{c}\begin{array}{c}\text { Number of } \\
\text { malaria } \\
\text { microscopy } \\
\text { tests in } 2004\end{array} \\
\end{array}$ & $\begin{array}{c}\text { Malaria } \\
\text { slide } \\
\text { positivity } \\
\text { rate }(\%) \\
\end{array}$ \\
\hline \multirow{2}{*}{ Tertiary } & 2 & 1800 & ND & 8700 & 51 \\
\hline & 2 & 80 & ND & 3251 & 23.4 \\
\hline \multirow{11}{*}{ Secondary } & 2 & - & ND & 566 & 83.3 \\
\hline & 2 & 12525 & 15 & 1109 & 84.2 \\
\hline & 2 & 790 & 15 & 2016 & 38.9 \\
\hline & 1 & - & ND & 1448 & ND \\
\hline & 2 & 349 & 14 & 953 & ND \\
\hline & 1 & 508 & 11 & 2350 & ND \\
\hline & 2 & - & ND & - & ND \\
\hline & 2 & 37 & ND & 205 & ND \\
\hline & 2 & 320 & 22 & 476 & ND \\
\hline & 1 & - & ND & - & ND \\
\hline & 1 & - & ND & - & ND \\
\hline Total & 22 & 16329 & & 21074 & \\
\hline
\end{tabular}


slides examined in the other four laboratories ranged from 37 in $\mathrm{BCH}$ to 1800 in $\mathrm{MMSH}$. The $\mathrm{TB}$ slide positivity rates in these laboratories ranged from $11 \%$ to $22 \%$.

Malaria microscopy workload was highest in MMSH and MAWSH laboratories, which examined 8,700 and 3251 malaria slides respectively in 2004. Malaria slides examined in MMSH constituted more than $40 \%$ of malaria slides examined in all the laboratories included in this assessment. The number of malaria slides examined in the other laboratories ranged from 205 in SML to 2,016 in WDL laboratories. The malaria slide positivity rates ranged from $23.4 \%$ to $84.2 \%$.

Implementation of integrated $T B$ and malaria microscopy QA scheme (2005-2007)

Integrating TB and malaria microscopy EQA scheme

The training workshop introduced participants to the principles and practice of quality assessments for $\mathrm{TB}$ and malaria and the feasibility of developing an integrated system for assessment of $\mathrm{TB}$ and malaria slides. However, evaluation of the impact of the training was not feasible. The integrated TB-malaria QA scheme was fully or partially achieved in four of the five laboratories. Full integration of QA was achieved in two laboratories (IDH and WDL), where microscopists were able to make available all the TB and malaria slides examined in their laboratories during supervisory visits. Partial integration was achieved in two other laboratories (GWZ and DBT) where microscopists did not store malaria slides during the baseline assessment period.

Assessment of the quality of TB and malaria microscopy results

TB slides were assessed on six occasions, while malaria slides were assessed on three occasions. Results of the baseline quality of TB microscopy from the five laboratories compared with results of the final assessment at 24 months showed that the concordance rate increased from $81.0 \%(\mathrm{~K}=0.353)$ to $91.0 \% \quad(\mathrm{~K}=$ 0.792). The concordance rate had a positive correlation with well-made sputum smears $(r=0.539)$ and staining $(r=0.963)$ (Table 2). Concordance rate of malaria microscopy from IDH and WDL, where full integration was achieved, increased from $69.2 \%$ at the baseline $(\mathrm{K}$ $=0.435)$, to $83.3 \%$ at the final assessment $(\mathrm{K}=0.667)$ in IDH and decreased from $100 \%$ to $83.3 \%$ in WDL. There was a similar positive correlation of concordant malaria microscopy results with well-made blood films $(\mathrm{r}=0.908)$ and good staining $(\mathrm{r}=0.963)$.

The proportion of false positive TB microscopy readings from all five laboratories combined decreased significantly $\left(\chi^{2}=59.8, \mathrm{P}<0.001\right)$ from $19.0 \%$ at the baseline to $1.8 \%$ at the final assessment. The false positivite rates of TB microscopy results decreased in four of the laboratories. There was also a decrease in the false positivite rate of malaria microscopy in IDH from $30.8 \%$ at the baseline to $0 \%$ at the final assessment. The false negative rate increased from $0 \%$ at the baseline to $3.6 \%$ at the final assessment due to the appearance of false negative results from IDH and DBT laboratories at the final assessment (Table 3).

Table 3. Results of LQAS and blinded rechecking of TB and malaria microscopy slides from five laboratories in Kano (2005-2006).

\begin{tabular}{|c|c|c|c|c|c|c|c|c|c|}
\hline \multicolumn{2}{|c|}{ Quality assessments } & \multicolumn{8}{|c|}{ TB microscopy } \\
\hline Laboratories & $\begin{array}{l}\text { Indices of } \\
\text { assessment }\end{array}$ & $\tilde{z}$ & 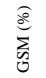 & $\frac{8}{5}$ & $\frac{\sigma}{z}$ & बi & $\frac{\mathrm{g}}{z}$ & 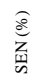 & $\frac{8}{\stackrel{s}{w}}$ \\
\hline \multirow{2}{*}{ IDH } & Baseline & 28 & 50 & 67.9 & 78.6 & 21.4 & 0 & 100 & 76 \\
\hline & Final & 15 & 46.7 & 40 & 86.7 & 0 & 6.7 & 66.7 & 100 \\
\hline \multirow{2}{*}{ WDL } & Baseline & 11 & 36.4 & 72.7 & 72.7 & 27.3 & 0 & 100 & 66.7 \\
\hline & Final & 15 & 73.3 & 80 & 93.3 & 0 & 0 & 100 & 100 \\
\hline \multirow{2}{*}{ GWZ } & Baseline & 22 & 40.9 & 45.5 & 95.5 & 4.5 & 0 & - & - \\
\hline & Final & 14 & 64.3 & 71.4 & 100 & 0 & 0 & 100 & 100 \\
\hline \multirow{2}{*}{ DBT } & Baseline & 27 & 18.5 & 22.2 & 96.6 & 3.7 & 0 & 100 & 96 \\
\hline & Final & 12 & 41.7 & 41.7 & 83.3 & 8.3 & 8.3 & 50 & 90 \\
\hline \multirow{2}{*}{ RAN } & Baseline & 12 & 50 & 41.7 & 33.3 & 66.6 & 0 & - & - \\
\hline & Final & $\mathrm{NA}$ & $\mathrm{NA}$ & $\mathrm{NA}$ & NA & $\mathrm{NA}$ & NA & $\mathrm{NA}$ & $\mathrm{NA}$ \\
\hline \multirow{2}{*}{$\begin{array}{l}\text { All centres } \\
\text { combined }\end{array}$} & Baseline & 100 & 38 & 48 & 81 & 19 & 0 & 100 & 80 \\
\hline & Final & 56 & 57.1 & 58.9 & 91.1 & 1.8 & 3.6 & 77.8 & 97.9 \\
\hline \multicolumn{2}{|c|}{ Quality assessments } & \multicolumn{8}{|c|}{ Malaria microscopy } \\
\hline Laboratories & $\begin{array}{l}\text { Indices of } \\
\text { assessment }\end{array}$ & $\tilde{z}$ & 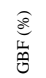 & $\frac{8}{5}$ & $\frac{\mathfrak{g}}{z}$ & बृ & $\frac{8}{z}$ & $\begin{array}{l}\stackrel{8}{z} \\
\text { 丞 }\end{array}$ & $\frac{c}{\frac{b}{w}}$ \\
\hline \multirow{2}{*}{ IDH } & Baseline & 13 & 46.2 & 69.2 & 69.2 & 30.8 & 0 & 100 & 55.6 \\
\hline & Final & 6 & 50 & 50 & 83.3 & 0 & 16.7 & 75 & 100 \\
\hline \multirow{2}{*}{ WDL } & Baseline & 13 & 61.5 & 84.6 & 100 & 0 & 0 & 100 & 100 \\
\hline & Final & 6 & 83.3 & 83.3 & 83.3 & 16.7 & 0 & 66.7 & 100 \\
\hline \multirow{2}{*}{ GWZ } & Baseline & $\mathrm{NA}$ & NA & $\mathrm{NA}$ & $\mathrm{NA}$ & NA & NA & $\mathrm{NA}$ & $\mathrm{NA}$ \\
\hline & Final & 6 & $\mathrm{NA}$ & 50 & 66.7 & 16.7 & 16.7 & 75 & 100 \\
\hline \multirow{2}{*}{ DBT } & Baseline & $\mathrm{NA}$ & $\mathrm{NA}$ & $\mathrm{NA}$ & $\mathrm{NA}$ & NA & NA & $\mathrm{NA}$ & $\mathrm{NA}$ \\
\hline & Final & 6 & 50 & 16.7 & 66.7 & 16.7 & 16.7 & 66.7 & 66.7 \\
\hline \multirow{2}{*}{ RAN } & Baseline & NA & NA & $\mathrm{NA}$ & $\mathrm{NA}$ & NA & NA & $\mathrm{NA}$ & $\mathrm{NA}$ \\
\hline & Final & $\mathrm{NA}$ & $\mathrm{NA}$ & $\mathrm{NA}$ & NA & $\mathrm{NA}$ & NA & NA & $\mathrm{NA}$ \\
\hline \multirow{2}{*}{$\begin{array}{l}\text { All centres } \\
\text { combined }\end{array}$} & Baseline & 26 & 53.8 & 76.9 & 84.6 & 15.4 & 0 & 100 & 77.8 \\
\hline & Final & 24 & 62.5 & 50 & 75 & 8.7 & 13 & 76.9 & 80 \\
\hline
\end{tabular}

KEY: NS = Number of slides sampled. GSM = Good sputum smears. GBF = Good blood film. GST = Good staining. $\mathrm{CON}=$ concordance rate. $\mathrm{FP}=$ false positivity rate. $\mathrm{SES}=$ Sensitivity. $\mathrm{SPE}=$ Specificity . NA $=$ not assessed. - Sensitivity and specificity not determined. 
There was an increase in the specificity of TB microscopy reading from all laboratories from $80 \%$ at the baseline to $97.9 \%$ at the final assessment while there was a corresponding decrease in sensitivity from $100 \%$ at the baseline to $77.8 \%$ at the final assessment (Table $3)$. At the final assessment, $100 \%$ specificity was achieved for TB microscopy results in four laboratories. A specificity of $100 \%$ for malaria microscopy was maintained in WDL throughout the study, while in IDH specificity of malaria microscopy results increased from $55.6 \%$ at baseline to $100 \%$ at the final assessment (Table 3).

\section{Discussion}

Timely and accurate diagnosis of TB and malaria and appropriate treatment are essential for reducing disease burden as well as transmission in the community $[15,16,17]$. The cost of implementing malaria drug policies based on combination therapy could be reduced considerably by improving the accuracy of diagnosis, which would target Artemisininbased Combination Therapy at those who need it and reduce over-consumption of antimalarial drugs by patients who do not have malaria [9].

The WHO has recommended the integration of malaria microscopy QA with that of other microscopically diagnosed communicable diseases [9], but there is no evidence for how this can be put into practice. There are no published data about the design and implementation of integrated TB-malaria QA systems. In this project, a QA for malaria microscopy was developed from, and systematically integrated into, the existing QA system for TB microscopy. Full or partial implementation of the integrated TB-malaria QA was achieved in four of the five selected laboratories.

In this system, TB and malaria microscopy results recording, slide storage, monitoring visits to the laboratories, LQAS slide sampling, the process of rechecking of both by two experienced microscopists, results analyses, and feedback mechanisms for communicating the performance of individual laboratories were combined for malaria and TB microscopy. The use of the same microscopists and microscopes and the provision of joint training and supervision for $\mathrm{TB}$ and malaria were feasible at the laboratory level.

The one-day refresher training of microscopists was insufficient to demonstrate improvement in their individual competency to identify $\mathrm{AFB}$ and malaria parasites in thin blood film. However, the training and integrated QA system may have improved the quality of
TB and malaria microscopy results from the five laboratories, particularly specificity. The average specificity of TB microscopy from the five laboratories increased from $80 \%$ to $97.9 \%$, and for the two laboratories undertaking malaria microscopy QA it increased from $76.0 \%$ and $66.7 \%$ to $100 \%$. The average specificity of malaria microscopy from the two laboratories increased from $77.8 \%$ to $80.0 \%$. The TB microscopy specificity of $96.8 \%, 98.9 \%$ and $100 \%$ were reported in Ethiopia [18] Argentina [19] and Tanzania [20]. Malaria microscopy specificity of $93.5 \%-98.3 \%$ was observed in ten laboratories on the Thai-Myanmar border of Thailand [21].

On average, the concordance rate of TB microscopy results increased from $81 \%$ at baseline to $91.0 \%$ at the final assessment. For malaria microscopy the concordance rate increased from $69.2 \%$ at the baseline to $83.3 \%$ at the final assessment in IDH, but decreased from $100 \%$ to $83.3 \%$ in the WDL laboratory due to $16.7 \%$ false positive results. Increases in the concordant $\mathrm{TB}$ and malaria microscopy results were positively associated with the ability of the laboratories to prepare and stain sputum smears and blood films for TB and malaria microscopy respectively. The concordance rates of TB microscopy achieved in Kano were similar to those obtained in other developing countries. Concordance rates of $\geq 97 \%$ in TB microscopy readings were reported in Malawi [22], Argentina [19] and southern Ethiopia [18] and $86.2 \%$ in malaria in South Africa [23].

The feasibility of integrating the QA system in the two laboratories was characterized by good motivation and commitment of staff in both laboratories as well as clear understanding of the purpose of the quality assessment at the start of the project. Laboratories where partial integration was achieved showed lack of motivation at the start of the project and appeared to perceive QA as a threat.

One of the limitations of the study was that one of the five laboratories provided $70 \%$ of the slides and only two of the five could be fully evaluated for full integration. Although the model and the QA monitoring team worked well at peripheral laboratory level, scaling up this integrated system to cover all laboratories in Kano State will be difficult. The laboratories in this study may not be truly representative so conclusions must be drawn with caution and the model will need testing in a variety of situations before generalisations can be made. There are no guidelines in the national or state health policy for the development and implementation of a malaria microscopy EQA system, 
so there are no resources or commitment to establish malaria QA either as stand-alone or integrated. The feasibility of such integration therefore requires a policy for its development and implementation at the state level.

Implementing an integrated TB and malaria QA scheme at the control programme level requires an integrated management system for both TB and malaria control programmes, in terms of administrative and technical responsibilities. The TB control programme had mechanisms to supply reagents and materials to these laboratories, so there is a potential for incorporating materials needed for malaria microscopy into this existing system. A laboratory central coordinating unit at the federal level would be helpful because it could be the focus of coordination between the TB and malaria control programmes and other programmes that rely heavily on quality of laboratory services. One of the major advantages of this integrated system is that it is not dependent on external funding. Rather it needs strong management and organization using internal resources. One of the big risks is that it depends on a well functioning TB QA programme.

This project demonstrated that it is feasible to integrate the training and QA system for TB and malaria microscopy and to achieve an improvement in the quality of both services. However, the integrated system needs to be tested in different settings to develop sound recommendations to guide the complex scalingup process.

\section{Acknowledgements}

This study was completed as part of a $\mathrm{PhD}$ programme sponsored by the Ford Foundation International. We are grateful to the personnel of the Kano state Ministry of Health and particularly to Dr Dayyabu Muhammad, Dr Amiru Imama Yola, Dr Nasir Mahmud, and the entire laboratory staff in the health facilities included in this project. Special thanks to Al-Muktar Yahuza, Saleh Idris Tudun-wada, Abdurrazaq Hamza, and Ado Garba Abubakar for their assistance in the collection of these data.

\section{References}

1. K-SEEDS (2005) Kano State Economic Empowerment and Development Strategy. March 2005. Available: www.kanostate.net. Accessed 7/5/2006.

2. NTBLCP, (2004): National Tuberculosis and Leprosy Control Programme (NTLCP) Workers' Manual 4th Edition, Federal ministry of Health Nigeria, Department of Public Health Abuja.

3. WHO (2005): World Malaria Report 2005, Roll Back Malaria May 2005

4. MOH, Kano (2004): Infectious Disease Surveillance Report. Kano State Malaria Control Programme. Ministry of Health, Kano State.

5. Paramasivan, C. N. Venkataraman, P. Vasanthan, J. S. Rahman, F. Narayanan P. R. (2003): Quality assurance studies in eight state tuberculosis laboratories in India Int. J. Tuberc. Lung Dis. 7(6):522-527

6. Hertzberg, M. S Mammen, . J. Mccraw, A. Nair S.C. and Srivastava A. (2006): Haemophilia 12, (Suppl. 3), 61-67

7. Plebani M. (2002): Charting the course of medical laboratories in a changing environment. Clinica Chimica Acta 319 (2002) 87-100 www.elsevier.com/locate/clinchim accessed 1/1/2008

8. FMOH (2005): Training Manual for Management of Malaria in Nigeria. Participants' Manual Federal Ministry of Health National Malaria and Vector Control Division Abuja, Nigeria. February 2005

9. WHO (2005): Malaria Light Microscopy. Creating a Culture of Quality. Report of WHO SEARO/WPOR Workshop on Quality Assurance For Malaria Microscopy. Kaula Lampur, Malaysia April 2005.

10. WHO (1998): Quality Systems for Medical Laboratories: Guidelines for Implementation and Monitoring. World Health Organization Regional Office for the Eastern Mediterranean. Alexandria, 1998

11. APHL, (2002): External Quality Assessment for AFB Smear Microscopy. Association of Public Health Laboratories, CDC, IUATLD, KCNV, RIT and WHO. Washington DC.

12. MOH Ghana (2001): Regional In-service Training (RIST) Programme for Laboratory Staff and Establishment of Nationwide Quality Control System. Ministry of Health Ghana. June 2001.

13. Salvakumar N., Murthy B. N, Prabhakaran E., Sivagamasundari S., Vasanthan S., Perumal M., govidaraju R., Chauhan L. S., Wares F., Santha T. and Narayana P. R. (2005): Lot Quality Assurance Sampling of Sputum Acid-Fast Bacillus Smear for Assessing Sputum Smear Microscopy Centres. Journal of Clinical Microbiology 43(2) 913 -15

14. IUATLD (2000): Technical guide. Sputum Examination for Tuberculosis by Direct Microscopy in Low Income Countries. (5ed) Paris. P1.

15. Maher D. (1999): Smear-Positive Pulmonary Tuberculosis: Good Clinical Management is Good Public Health. Africa Health 21(4): 6-9.

16. Paramasivan, C. N. Venkataraman, P. Vasanthan, J. S. Rahman, F. Narayanan P. R. (2003): Quality assurance studies in eight state tuberculosis laboratories in India Int. J. Tuberc. Lung Dis. 7(6):522-527

17. Barnish G, Bates I, Iboro J (2004): Newer drug combination for malaria may be impractical unless diagnostic accuracy can be improved. British Medical Journal (Editorials) (328) 1511-2

18. Shargie E. B, Yassin M. A. Lindtjorn B. (2005): Quality control of sputum microscopic examinations for acid fast bacilli in southern Ethiopia Ethiop. J Health Dev.;19(2)

19. Kusznierz, G. F. Latini, O. A. and Sequeira M. D (2004): Quality assessment of smear microscopy for acid-fast bacilli in the Argentine tuberculosis laboratory network, 1983-2001 Int J Tuberc Lung Dis 8(10):1234-1241

20. Basra, D. Matee M. I. N. McNerney R. Quality assessment of sputum smear microscopy for the detection of acid fast bacilli in peripheral health care facilities in Dar es Salaam, Tanzania East African Med. J. 2006 83(6): 306-310

21. Hemme F. and Gay F. (1998): Internal quality control of the malaria microscopy diagnosis for 10 laboratories on the ThaiMyanmar border. South Asian J Trop Med Public Health 29 (3): 529-36

22. Mundy C. J. F. Harries, A. D. Banerjee, A. Salaniponi, F. M. Gilks, C. F. Squire S. B. (2003): Quality assessment of sputum 
transportation, smear preparation and AFB microscopy in a rural district in Malawi Int. J. Tuberc. Lung Dis. 7(6):516-521

23. Dini L. and Frean J. Quality assessment of malaria laboratory diagnosis in South Africa (2003): Transactions Of The Royal Society Of Tropical Medicine And Hygiene 97, 675-677.

Corresponding Author: Faruk Sarkinfada, Department of Medical Microbiology and Parasitology, Faculty of Medicine, Bayero University, P. M. B. 3011, Kano, Nigeria

Email: fsarkinfada@yahoo.co.uk

Conflict of interest: No conflict of interest is declared. 